\title{
Erratum to: A prospective study of the cumulative incidence and course of restless legs syndrome in de novo patients with Parkinson's disease during chronic dopaminergic therapy
}

\author{
Elena Marchesi $^{1}$ - Anna Negrotti ${ }^{1} \cdot$ Monica Angelini $^{1} \cdot$ Matteo Goldoni $^{3}$. \\ Giorgia Abrignani $^{1} \cdot$ Stefano Calzetti $^{1,2}$
}

Published online: 19 February 2016

(C) Springer-Verlag Berlin Heidelberg 2016

\section{Erratum to: J Neurol \\ DOI 10.1007/s00415-015-7937-7}

The original version of this article unfortunately contained a mistake. First and last names of the authors were interchanged. The correct author names are given below. The original article has been corrected.

Elena Marchesi, Anna Negrotti, Monica Angelini, Matteo Goldoni, Giorgia Abrignani and Stefano Calzetti

The online version of the original article can be found under doi:10.1007/s00415-015-7937-7.

Stefano Calzetti

stefano.calzetti@unipr.it

1 Department of Emergency and Medical Speciality Area, Neurology Unit, Azienda Ospedaliero-Universitaria of Parma, Parma, Italy

2 Department of Clinical and Experimental Medicine, Headache, Movement Disorders and Cerebrovascular Diseases Unit, University of Parma, Parma, Italy

3 Department of Clinical and Experimental Medicine, Laboratory of Industrial Toxicology, University of Parma, Parma, Italy 